\title{
Impairment Evaluation toward QKD Integration in a Conventional 20-Channel Metro Network
}

Slavisa Aleksic, Florian Hipp, Dominic Winkler, Andreas Poppe, Bernhard Schrenk and Gerald Franzl

(C) 2015 The Optical Society of America (OSA). This is an authors' copy of the work. It is posted here by permission of OSA for your personal use. Not for redistribution. The definitive version will be published in the proceedings of the Optical Fiber Communication Conference and Exposition (OFC 2015), Los Angeles, CA, March 2015. 


\title{
Impairment Evaluation toward QKD Integration in a Conventional 20-Channel Metro Network
}

\author{
Slavisa Aleksic ${ }^{1}$, Florian Hipp ${ }^{2}$, Dominic Winkler ${ }^{1}$, \\ Andreas Poppe $^{2}$, Bernhard Schrenk ${ }^{2}$ and Gerald Franzl ${ }^{1}$ \\ ${ }^{1}$ Institute of Telecommunications, Vienna University of Technology, Vienna, Austria \\ ${ }^{2}$ Safety \& Security Department, AIT Austrian Institute of Technology GmbH, Vienna, Austria \\ Phone: +43158801 38831, eMail: slavisa.aleksic@tuwien.ac.at
}

\begin{abstract}
Integration of QKD in telecom infrastructures raises noise issues not present in systems using dedicated fibers. Experiments on a 3-node metro network indicate that integration is possible when allocating broadband quantum channels in the O-band.
\end{abstract}

OCIS codes: $060.5565,270.5568$

\section{Introduction}

Current commercially available QKD systems generally presume dedicated point-to-point fiber links to avoid high levels of noise in the quantum channel by preventing any interaction between strong classical telecommunication channels and weak quantum channels. Integrating classical data channels and quantum channels within the same fiber is referred to as coexistence scheme, which is in principle possible [1-4]. This integration is economically promising because it saves the costs for installing/leasing additional fibers for QKD. However, in the coexistence scheme, the optical noise power in a quantum channel should be below 1 million photons/(s.nm) [3] to not severely impact the performance of a typical QKD system using the prepare and measure QKD schemes and BB84 protocol. Limiting the number of conventional WDM channels to four and reducing the launch-power far below the standardized levels, secret key rates below $1 \mathrm{Mbps}$ for distances up to several tens of kilometers are achievable [2]. Much higher key rates are theoretically possible when using more complex setups (e.g. multicarrier transmission analyzed in [5]). However, the restrictions on signal powers and the number of WDM channels are hardly achievable in practical WDM metro network installations. In this paper, we focus on the impairments introduced by a large number of strong classical signals as commonly used in the field, aiming to find a wavelength range that best suits quantum channels without the need to heavily constrain the classical WDM system.

\section{Integration of Quantum Key Distribution in Metropolitan Area Networks}

To realize the coexistence scheme, where the quantum channel, the key distillation channel and all classical communication channels are transmitted over the same fiber, demands so called QKD combiner/separator to sensibly add/drop/bypass the weak quantum channels. In [3] we show that scattering effects in the fiber medium, mainly Raman scattering, poses one of the major sources of the noise and that the QKD wavelength should preferably be chosen far below the wavelength of the noise-inducing data channels, e.g. in the O-band. To enable a reliable exchange of qubits in presence of strong classical signals an effective noise filtering in the O-band is essential. A cascade of two band multiplexers/demultiplexers (WDM couplers/splitters) is used as shown in Fig. 1, because with a single band

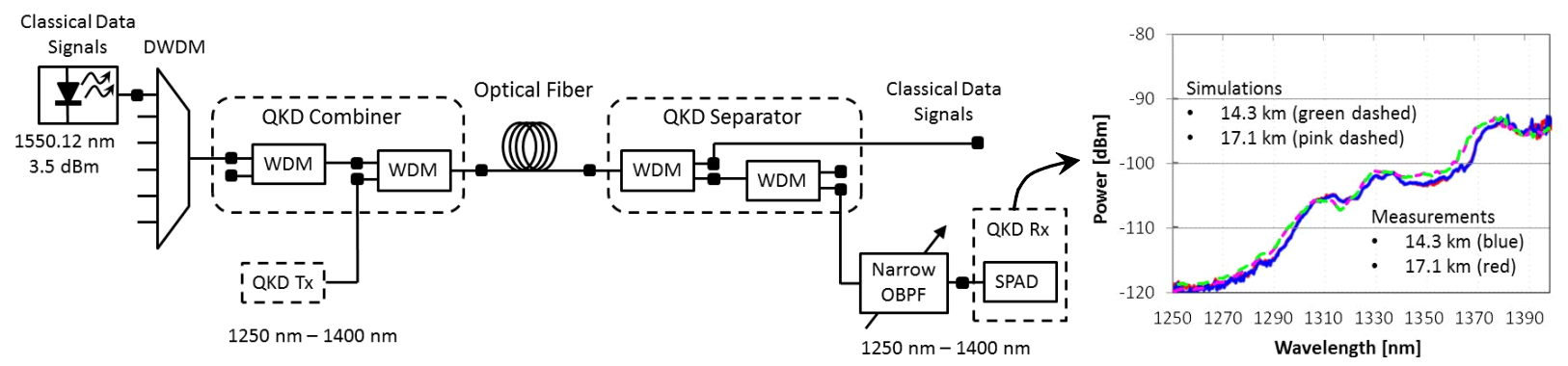

Fig. 1. Experimental and simulation setup for analyzing the influence of forward Raman scattering on integrated QKD systems in metropolitan area networks. Inset measurement and simulation results for $14.3 \mathrm{~km}$ and $17.1 \mathrm{~km}$ of standard single mode fibers (SSMF). 
filter sufficient band separation could not be achieved. Additionally, a narrow-band filter $(0.1 \mathrm{~nm}$ to $1 \mathrm{~nm})$ matched to the ability of different QKD systems to suppress in-band noise photons is required to further reduce the noise level. The experimental setup from Fig. 1 is used to analyze the combining/separating of quantum and classical channels and also to evaluate the influence of Raman scattering. As noise levels below $-90 \mathrm{dBm}$ can hardly be measured with conventional optical spectrum analyzers (OSA), we decided to count the noise photons using a single-photon avalanche photo diode (SPAD). A narrow coherent $1550.12 \mathrm{~nm}$ CW laser source with $3.5 \mathrm{dBm}$ optical output power is used as Raman pump. The measured forward Raman scattering in SSMF at lengths of $14.3 \mathrm{~km}$ and $17.1 \mathrm{~km}$ is shown inset in Fig. 1 together with simulation results (dashed lines). At $1250 \mathrm{~nm}$ the noise level is as low as $-120 \mathrm{dBm}$ and increases toward longer wavelengths. Above $1370 \mathrm{~nm}$ the optical noise power raises above $-100 \mathrm{dBm}$, which prohibits reliable exchange of qubits at longer wavelengths. Experimental and simulation results fit very well over the wavelength range of interest, i.e., between $1250 \mathrm{~nm}$ and $1400 \mathrm{~nm}$.

\section{Characterization of QKD Integration}

To analyze the performance of QKD integration in the metropolitan area we first configured a 3-node commercial DWDM system (Wave Star OLS400G) to provide 20 DWDM channels (classical data channels) as depicted in Fig. 2. The 20-channel DWDM signal generated in node 1 and amplified by an booster Erbium-doped fiber amplifier (EDFA) is tapped off using a 99/1 optical coupler. Cascaded 1310/1550 far-wavelength-division-multiplexers (FWDMs) are used for combining and separating quantum channels with classical data channels. Measured transmission and reflection curves for FWDM components and quantum efficiency of the SPAD are shown inset. Note that the SPAD is used to count noise photons within the O-band, while the spectrum above $1410 \mathrm{~nm}$ is measured using an OSA.

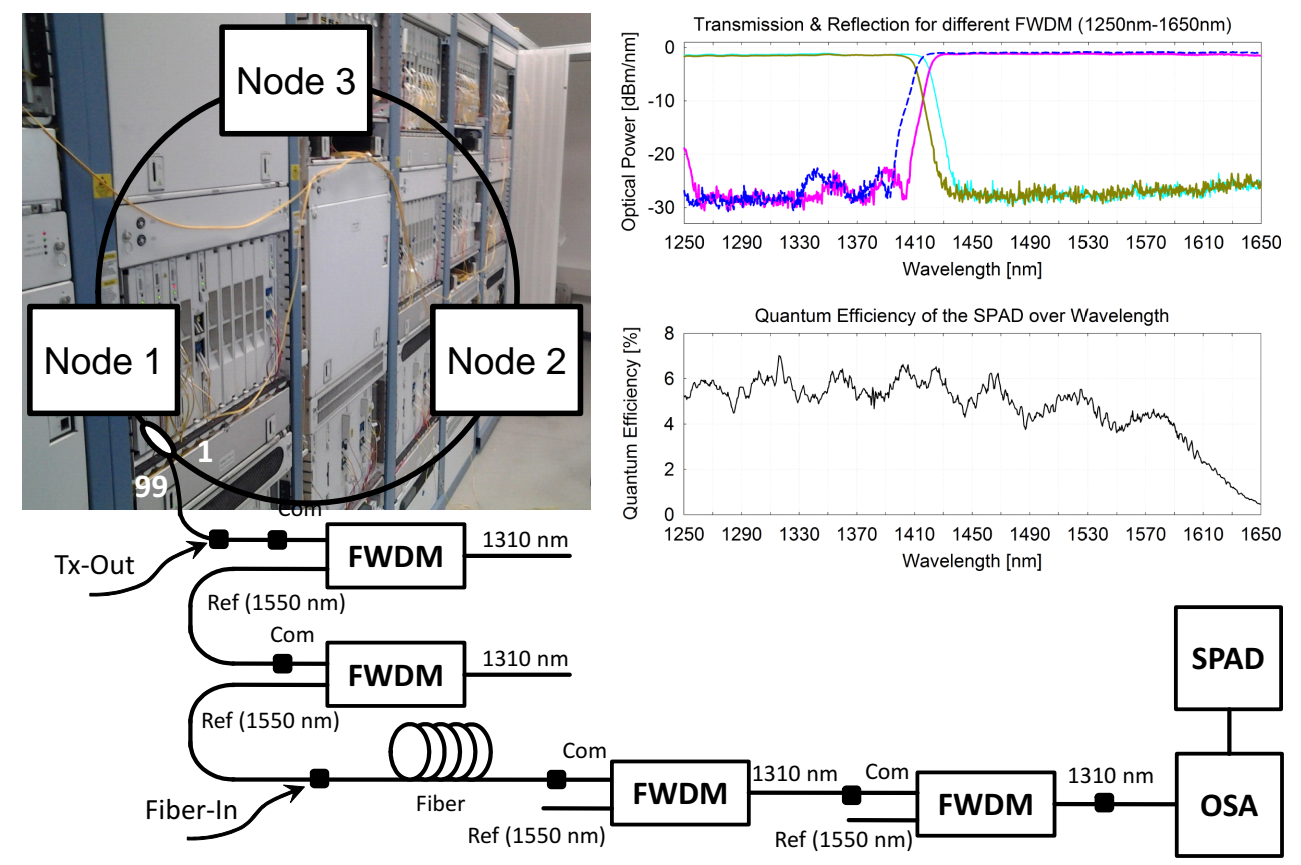

Fig. 2. Experimental setup for characterization of QKD integration in metropolitan area networks. Inset measured transmission and reflection curves for different FWDM components used to combine/separate quantum and classical channels as well as measured quantum efficiency of the single photon avalanche photodiode (SPAD) used to count noise photons below $1410 \mathrm{~nm}$.

To reflect the variety of installed fiber types, we choose standard (ITU-T G652-B) and low-water-peak single mode fibers of different age and length. Additionally, to address the influence of connectors and splices, we consider a $27 \mathrm{~km}$ long fiber made of two pieces ( $14 \mathrm{~km}$ and $13 \mathrm{~km}$ ) that are connected with each other by connectors. As can be seen from Fig. 3a, the noise within the O-band is mainly caused by the forward Raman scattering. A noise level below 1 million photons/(s.nm) has been obtained up to $1310 \mathrm{~nm}$, for all fiber types and lengths considered. The difference in noise levels between the considered fibers lies within a range of $10 \mathrm{~dB}$. This emphasizes the need for characterization of the installed fibers prior to considering QKD integration. Coexisting strong signals within the C-band cause a reduction of the useable wavelength range for about $60 \mathrm{~nm}$ when comparing to the single channel measurement shown in Fig. 1. 

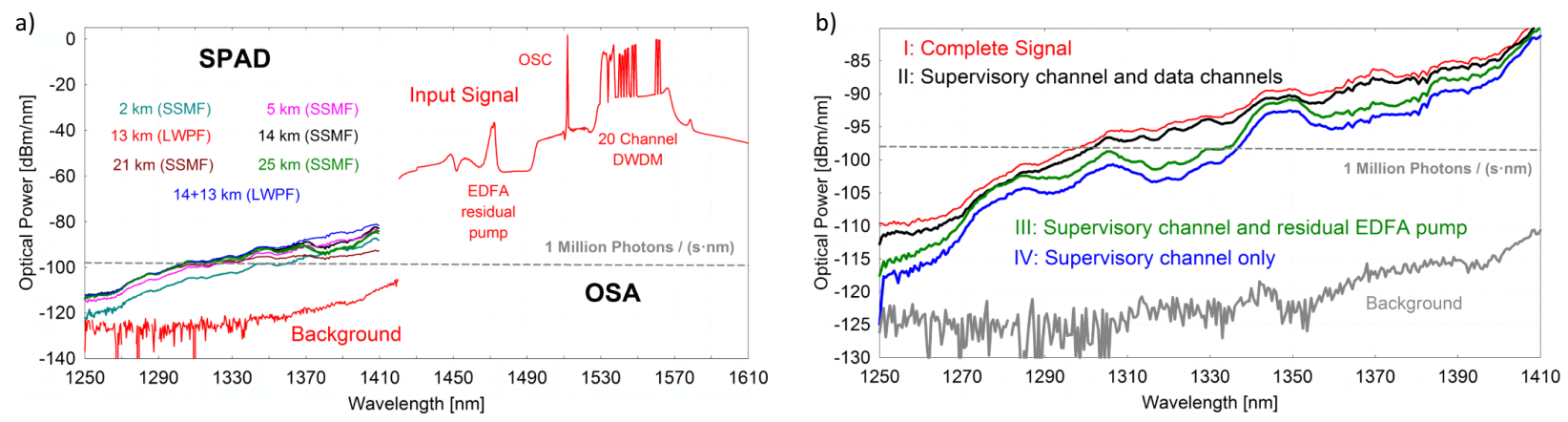

Fig. 3. Forward Raman scattering: a) noise power level within the O-band caused by the strong classical signals in the C-band of a conventional DWDM system in operation. Results are shown for different fiber types and lengths. Also background noise of the signal before transmission is shown (red line). b) Contributions of signals I - IV to the noise in the O-band.

To assess the contributions of the data channels, the optical supervisory channel (OSC) and the residual EDFA pump to the noise in the O-band, we carried out measurements with the complete signal (case I), with OSC and data channels without EDFA pump (case III) and with OSC and EDFA pump (case III) OSC only (case IV). The input signals used to characterize the main cause of the noise are obtained by using appropriate filters to split up the signal generated by node 1 into signals I to IV. The corresponding results for a fiber length of $14 \mathrm{~km}$ are shown in Fig. $3 \mathrm{~b}$. The blue curve represents the measured photon noise level in the O-band when the DWDM signal and the residual EDFA pump are removed (case IV). Here, the background noise noise is only due to the OSC signal. Even though OSC is only a single channel, it has a significant influence on the noise generated in the O-band since it is allocated at least $20 \mathrm{~nm}$ closer to the quantum channel than the data channels. To obtain the green curve representing case III, we filter out the DWDM signal. Here, an increase of the photon noise by about $2 \mathrm{~dB}$ in comparison to case IV has been observed, which is mainly due to the EDFA's amplified spontaneous emission (ASE) noise that is present in signal III and completely removed from signal IV. Finally, we filter out the residual EDFA pump only in order to assess its influence. The corresponding noise spectrum is shown by the black curve (case II), which is close to the red curve (case I - entire signal). This result indicates that the influence of the residual EDFA pump signal can be neglected. Even though it is spectrally closer to the O-Band than the OSC, its low power (about $-40 \mathrm{dBm}$ ) does not suffice to generate many scattered photons in the O-band. The 20-channel DWDM signal contributes by up to $5 \mathrm{~dB}$ to the photon noise. This significant contribution varies with wavelength, the number of classical channels and their power levels.

\section{Conclusions}

An integration of QKD channel into existing telecommunication networks boils down to the question whether a QKD scheme can be found that withstands the high noise levels originating from strong classical data signals. The presented measurements show that an elaborated positioning of classical and quantum channels can reduce noise to a tolerable level for a QKD system. However, key rate and user scaling still remain challenging issues. Possible approaches to tackle these problems are filtering in the time and wavelength domain. The latter can be achieved with standard equipment, as shown in the presented approach that propose the allocation of quantum channels in the O-band, which also simplifies the bypassing of active network equipment such as optical nodes and amplifiers as well as multiplexing and switching quantum signals toward QKD networks.

Acknowledgment: The Austrian FFG supported this work through the FIT-IT project QKD-Telco no. 835926.

\section{References}

1. P. Eraerds, et al., in New Journal of Physics, vol. 12, no. 6, 2010, paper 063027.

2. K. A. Patel, et al., in Physical Review X, vol. 2, 2012, paper 041010.

3. S. Aleksic, et al., in ICTON 2013, Cartagena, Spain, June 23-27, 2013, paper We.B1.3.

4. T. E. Chapuran, et al., in New Journal of Physics 13, 2009, paper 105001.

5. M. Cvijetic, et al., ICTON 2013, Cartagena, Spain, June 23-27, 2013, paper We.B1.2. 